\title{
Process Evaluation of a Combined Lifestyle Intervention for Community-Dwelling Older Adults: ProMuscle in Practice
}

\author{
Ellen J. I. van Dongen, PhD, ${ }^{1}$ Esmée L. Doets, PhD, ${ }^{1}$ Lisette C. P. G. M. de Groot, PhD, ${ }^{2}$ \\ Berber G. Dorhout, $\mathrm{MSc}^{2}$ and Annemien Haveman-Nies, $\mathrm{PhD}^{2,3}$
}

'Food, Health \& Consumer Research, Wageningen Food \& Biobased Research, Wageningen, The Netherlands. ${ }^{2}$ Division of Human Nutrition and Health, Wageningen University \& Research, Wageningen, The Netherlands. ${ }^{3}$ Chair group Consumption and Healthy Lifestyles, Wageningen University and Research, Wageningen, The Netherlands.

*Address correspondence to: Annemien Haveman-Nies, PhD, Chair group Consumption and Healthy Lifestyles, Wageningen University and Research, P0 Box 17,6700 AA Wageningen, The Netherlands. E-mail: annemien.haveman@wur.nl

Received: September 25, 2019; Editorial Decision Date: February 19, 2020

Decision Editor: Suzanne Meeks, PhD

\begin{abstract}
Background and Objectives: The ProMuscle in Practice intervention combines resistance exercise training and dietary protein intake for community-dwelling older adults, implemented by health care professionals (HCPs). This study aimed to evaluate implementation and context of this intervention in Dutch health care practice.

Research Design and Methods: We conducted a randomized controlled multicenter intervention study in 5 Dutch municipalities. Eighty-two older adults received the 12-week intensive support intervention (resistance exercise training and individual dietary counseling) and the optional 12-week moderate support intervention (resistance exercise training and a nutrition course). Mixed method data were collected from both participants and HCPs $(n=37)$ on process indicators recruitment, dose received, acceptability, fidelity, applicability, and context.

Results: Overall, the intervention was feasible to implement and accepted by participants and HCPs. About two thirds of participants continued with the moderate support intervention after the first 12 weeks. The mean dose received for the training sessions was $83.6 \%$ in the intensive intervention, $63.6 \%$ in the moderate intervention, $>90 \%$ for individual dietitian consultations, and $76.8 \%$ for the nutrition course. The intensive support intervention was implemented with high fidelity, whereas for the moderate support intervention resistance exercise trainings varied in implementation between exercise providers.

Discussion and Implications: A combined resistance exercise training and dietary protein intervention for communitydwelling older adults can be successfully implemented in practice. Well-tailored interventions, intensive supervision by skilled HCPs, social aspects, fidelity, and fit within real-world settings appeared essential for successful implementation. These elements are important for continuous intervention optimization to accomplish broader and successful implementation.
\end{abstract}

Keywords: Dietary protein, Nutrition, Resistance exercise training, Implementation

Age-related decline in muscle mass and strength contributes to a decrease in physical functioning (Morley, Baumgartner, Roubenoff, Mayer, \& Nair, 2001) and can reduce quality of life (Cruz-Jentoft et al., 2010). These declines can be counteracted by combining resistance exercise training and protein supplementation (Cermak, Res, de Groot, Saris, \& 
van Loon, 2012; Hou et al., 2019), and thus implementing effective interventions combining these aspects in practice is needed (Cruz-Jentoft et al., 2014). Implementing such complex lifestyle interventions successfully in real-world settings should consider whether the setting and the organizational and professional context influence the intervention and its outcomes (Durlak \& DuPre, 2008; Moore et al., 2015). Tailoring interventions to the local context can increase intervention effectiveness (Craig et al., 2013), but to sustain implementation it should be reported what was tailored and why (Moore et al., 2015). Besides focusing on organizational and implementer factors, participant engagement with and acceptability of the intervention is also important for intervention success (Moore et al., 2015). Most randomized controlled trials on intervention effectiveness report attendance or intervention compliance (i.e., Daly et al., 2014; Tieland et al., 2012; Vikberg et al., 2019), but seldom report on acceptance, context, or the underlying intervention theory. To promote broader adoption of effective interventions, understanding what works, for whom, and under what conditions is necessary (Moore et al., 2015; Salter \& Kothari, 2014).

An efficacious combined resistance exercise training and protein supplementation intervention (Tieland et al., 2012) was previously adapted to ProMuscle in Practice, to fit in the practice setting (van Dongen et al., 2017). A multicenter, real-life study has shown effectiveness of the ProMuscle in Practice intervention in improving muscle strength, body composition, and physical functioning when implemented in a real-life setting (van Dongen, Haveman-Nies, Doets, Dorhout, \& De Groot, 2020), but these effects vary per setting and intervention period. To obtain insight into how the ProMuscle in Practice intervention produced these results, a process evaluation is performed to assess implementation, and to identify mechanisms of impact or contextual factors that can explain variance in effectiveness outcomes (Craig et al., 2008; Moore et al., 2015). Therefore, the aim of this study was to investigate implementation and context of the ProMuscle in Practice intervention for communitydwelling older adults in Dutch health care practice.

\section{Methods}

\section{Study Design and Setting}

The ProMuscle in Practice randomized, controlled, multicenter intervention study was conducted between September 2016 and November 2018 in five municipalities in the Netherlands. The study duration was 36 weeks in municipalities Apeldoorn and Ede, and 52 weeks in Epe, Ermelo/Putten, and Harderwijk. The study protocol was approved by the Wageningen University Medical Ethics Committee, the Netherlands, and all participants gave their written informed consent before participation. The study was registered with The Dutch Trial Register (NTR, number 6038) on August 30, 2016. Study design, and intervention theory and description have been reported in detail elsewhere (van Dongen et al., 2018).

\section{Study Population}

The study population consisted of community-dwelling older adults ( 65 years or older) who were mainly recruited through local media. During screening, researchers evaluated whether interested persons met one of the two inclusion criteria: (a) being prefrail or frail based on the Fried frailty criteria (Fried et al., 2001) or (b) experiencing difficulty in selected activities of daily living (ADL) tasks and not performing resistance exercises $\geq 2$ times a week. If so, the general practitioner (GP) checked the specified exclusion criteria mainly regarding the presence of specific medical conditions (van Dongen et al., 2018). After inclusion, participants were randomly allocated to the intervention or control group at each location. This article focuses on the intervention group only as they received the ProMuscle in Practice intervention.

\section{Intervention}

The intervention consisted of a 12-week intensive support intervention and a subsequent voluntary 12 -week moderate support intervention, providing postintervention support (van Dongen et al., 2018).

\section{Intensive Support Intervention}

The intensive support intervention was based on previous studies (Tieland et al., 2012; van Dongen et al., 2017) and included a resistance exercise component and a dietary protein component, implemented by physiotherapists and dietitians of local care organizations. The care organizations that adopted the intervention were recruited mainly through existing networks of project partners. The paramedical departments of these organizations inquired who of the health care professionals (HCPs) were willing to implement the intervention. These HCPs received a 1.5$\mathrm{hr}$ training session by one of the researchers (including explanation of the background, intervention content, and materials) and detailed implementation manuals before the intervention started. In week 6 of the intervention, the HCPs at each location had a $1-\mathrm{hr}$ joint peer discussion to compare and discuss experiences with intervention implementation and formulate solutions to potential problems. During the intervention, the HCPs could contact the researchers when needed, and researchers checked up on the HCP on a nonstructural basis.

Resistance exercise component.-Participants performed 1-hr progressive resistance exercise twice a week, supervised by physiotherapists, in groups (4-6 participants). Each training consisted of a warm-up, five strength exercises, and a warm-down. Leg exercises training intensity was based on 
personal maximum strength tests (1 repetition maximum $[1 \mathrm{RM}])$, and these exercises were performed progressively. Physiotherapists used a maximum strength test in week 6 to further tailor training intensity. Physiotherapists motivated participants to perform the exercises correctly and progressively, built participants' confidence in performing resistance exercise, promoted social cohesions, and informed participants on the moderate support intervention.

Dietary protein component.-Dietitians counseled participants to achieve an intake of at least $25 \mathrm{~g}$ of protein per main meal, based on 3-day food diaries, and provided tailored advice to participants during a $30-\mathrm{min}$ intake consultation before the intervention started. Participants received mainly dairy-based protein-rich foods to incorporate in their diet, in agreement with participants' preferences. The dietitian was present to answer questions when participants first received the foods, and held individual evaluation consultations with participants in week 6 to potentially adjust the dietary advice and to discuss the continuation with consuming protein-rich foods after the intensive intervention. Dietitians monitored food consumption compliance through a weekly checklist completed by participants and discussed barriers and facilitators for increasing protein intake.

\section{Moderate Support Intervention}

The aim of this follow-up intervention was to guide participants toward behavior maintenance concerning resistance exercise and a protein-rich diet. This optional program started directly after the intensive support intervention and was designed by the municipal health service in collaboration with local organizations and municipalities. Approximately 3 weeks before the start, participants received a leaflet including information on the moderate support intervention and information about (home) exercise and protein-rich foods. Exercise groups (twice a week) were initiated at local gymnastic clubs, fitness centers, or physiotherapy practices, and a nutrition course was organized by the municipal health service. All exercise trainers offered group-based exercise sessions including strength exercises described in a general manual, but the type of exercises and professional guidance differed per location. Each nutrition course was implemented by a health promotor and dietitian. The course included five workshops, in which participants interacted, prepared protein-rich dishes (breakfast, lunch, and dinner), and visited a supermarket. Additionally, participants received a 2-3 monthly program newsletter through E-mail.

\section{Data Collection and Outcomes}

\section{Background Characteristics}

Participant background characteristics were assessed through a questionnaire at baseline, including questions on age, education level, living situation, care use, morbidities (Lutomski et al., 2013), current physiotherapist or dietitian guidance, and history of physical activity.

\section{Process Evaluation}

The process evaluation was informed by the Medical Research Council (MRC) guidelines for process evaluation (Moore et al., 2015) and the Conceptual Framework for Implementation Research (Proctor et al., 2011; Proctor et al., 2009). We collected mixed-method data from participants and HCPs on process indicators related to the domains implementation and context of the MRC framework (Moore et al., 2015); see Table 1 for details. For implementation, we focused on process indicators recruitment, reach, dose received, acceptability, fidelity, and applicability (Linnan \& Steckler, 2002; Moore et al., 2015; Proctor et al., 2011; Saunders, Evans, \& Joshi, 2005; Wang, Moss, \& Hiller, 2006). Context includes contextual aspects that influenced implementation or effectiveness (Durlak \& DuPre, 2008; Moore et al., 2015), which are described in the implementation results section. E. J. I. van Dongen (MSc-level researcher) conducted semistructured interviews with $37 \mathrm{HCPs}$ (through telephone, mean duration $43 \pm 11 \mathrm{~min}$ ) and 16 participants (face-to-face in their own home, mean duration $35 \pm 10 \mathrm{~min})$. All HCPs were invited for an interview (one physiotherapist declined due to personal reasons), and a convenience sample of participants was invited. The interview questions were based on guides pretested in the pilot study (van Dongen et al., 2017), and follow-up questions were used as needed.

\section{Data Analysis}

Quantitative data were analyzed using SPSS, version 23. Differences between background characteristics of the drop-outs and completers were tested using independent samples $t$ tests, $\chi^{2}$ tests, or Fisher's exact test. Descriptive statistics were used to analyze acceptability and dose received, and data were presented in mean and standard deviation, or frequency and percentage. Qualitative data were analyzed using an inductive approach (Thomas, 2006) in Atlas.ti, version 8. All interviews were audiotaped and transcribed verbatim. All transcripts were checked with the audio recording before analysis to remove transcribing errors and read in detail to familiarize with the content. Transcripts of HCP interviews were analyzed by E. J. I. van Dongen, and transcripts of participants were analyzed by a research assistant (Lilian Brouwer). Overarching categories were derived from the specified process indicators of Table 1, and for professionals also within their specified tasks [supplementary file of van Dongen et al., (2018)], lower-level categories were created based on the raw data. After initial coding, overlapping categories were combined and refined, and the most important themes are discussed in the Results section. 
Table 1. Overview of Process Indicators, Definitions, and Data Collection Within the Process Evaluation of ProMuscle in Practice for the Intensive Support Intervention and Moderate Support Intervention

Process indicator

Definition

Methods-intensive support intervention

Methods-moderate support intervention

Recruitment

Procedures used to attract participants

(Linnan \& Steckler, 2002)

Reach

Extent to which intended audience comes into contact with the intervention (Moore et al., 2015)

Dose received

Quantity of the intervention that was implemented and the extent to which participants actively engaged in intervention activities (Moore et al., 2015)

Acceptability

Extent to which the participants and $\mathrm{HCP}^{\mathrm{a}}$ were satisfied with the intervention (Proctor et al., 2011; Saunders et al., 2005)

Fidelity

Extent to which the intervention was implemented as planned (Linnan \& Steckler, 2002; Moore et al., 2015; Nutbeam, 1998; Proctor et al., 2011)

\section{Applicability}

Extent to which the intervention fitted in the real-world setting (Wang et al., 2006)
- Project logbook

- Registration forms

- Participant questionnaire T0 (incl. reasons to participate)

- Project logbook

- Registration forms

- Project logbook

- Registration forms

- Attendance lists of training sessions and dietitian consultations

- Registration forms on training intensity and topics discussed within dietitian consultations

- Participant questionnaire $T 0^{\mathrm{b}}$, incl. motivation to participate on Likert-type scale 1 (not motivated at all) to 5 (very motivated), reasons for participation

- Participant questionnaire T1 ${ }^{\mathrm{c}}$, incl. general acceptability score (1-10, higher score is higher acceptability), satisfaction with specific aspects of diet and exercise intervention (Likert-type scale 1 [not satisfied at all] to 5 [very satisfied]), and open questions on intervention acceptability

- Semistructured interviews with HCP at T1-18 physiotherapists/interns, 8 dietitians, incl. motivation to implement the program (1-10, higher score is higher motivation), questions on intervention acceptability and general acceptability score (1-10, higher score is higher acceptability)

Focused on whether the intervention was implemented as planned according to the structured manuals

- Semistructured interviews with HCP at $T 1$, incl. questions on whether, how and why they deviated from the intervention protocol

- Registration forms of training intensity and dietitian consultations

- Structured observations

- Project meetings minutes

- Semistructured interviews with HCP at $T 1$, incl. questions on whether the intervention fitted their regular working procedures
- Attendance lists of training sessions and nutrition course sessions

- Participant questionnaire T1, incl. motivation to participate on Likert-type scale 1 (not motivated at all) to 5 (very motivated)

- Participant questionnaire $T 2^{\mathrm{d}}$, incl. general acceptability score (1-10, higher score is higher acceptability), satisfaction with specific aspects of diet and exercise intervention (Likert-type scale 1 [not satisfied at all) to 5 [very satisfied]), open questions on intervention acceptability

- Semistructured interviews with participants at $T 2$ focused on the moderate support intervention $(n=4$ per municipality (total $N=16$ ), both males and females, including participants who participated in none, one or more of the intervention components)

- Semistructured interviews with HCP at T2-9 trainers, 2 nutrition course leaders, incl. questions on motivation to implement the program $(1-10$, higher score is higher motivation), intervention acceptability and general acceptability score (1-10)

Focused on what was actually implemented and how the intervention as described in the general manual was adapted to local context

- Semistructured interviews with HCP at $T 2$, incl. questions on intervention content and adaptations to local context

- Structured observations

- Project meetings minutes

- Semistructured interviews with HCP at $T 2$, incl. questions on whether the intervention fitted their regular working procedures 
Table 1. Continued

Process indicator

Definition

Context

Larger physical, organizational, social environment that could influence intervention implementation (Moore et al., 2015) and intervention outcomes (Durlak \& DuPre, 2008)
Methods-intensive support intervention

- Participants questionnaire T1, incl. questions on whether they followed a diet or participated in other exercise programs

- Semistructured interviews with HCP at $T 1$, incl. question on contextual factors that either facilitated or hindered intervention implementation

- Project logbook

- Project meeting minutes

- Semistructured interview with project coordinator $(n=1)$ that initiated adoption of part of the intervention
Methods-moderate support intervention

- Participants questionnaire T2, incl. questions on whether they followed a diet or participated in other exercise programs

- Semistructured interviews with participants at T2, incl. questions on reasons for (non)participation

- Semistructured interviews with HCP at $T 2$, incl. question on contextual factors that either facilitated or hindered intervention implementation

- Project logbook

- Project meeting minutes

- Semistructured interviews with project coordinators $(n=2)$ that initiated adoption of part of the moderate support intervention

${ }^{a} \mathrm{HCP}=$ health care professionals.

${ }^{\mathrm{b}} \mathrm{T} 0$ - baseline.

'T1-Week 12, directly after the intensive support intervention.

dT2-Week 24, directly after the moderate support intervention.

\section{Results}

\section{Recruitment and Reach}

Of the 296 screened older adults, 192 were initially included in the study by the GP (Supplementary Figure 1). Participants were mainly excluded because they were too fit, too active, or because they met one or more of the exclusion criteria related to diseases. Twenty-four participants withdrew between randomization and baseline measures, starting the baseline measures with 168 individuals, of which 82 intervention group participants. Main reasons for participation were contribution to science $(n=56)$, personal interest $(n=53)$, and personal improvement $(n=44)$.

Table 2 shows baseline characteristics of the total intervention group, and of the intervention participants who completed the trial versus those who dropped out. The mean age was 74.7 years, $37.8 \%$ were male, and $50 \%$ were nonfrail. The 11 drop-outs did not significantly differ from the completers in baseline characteristics, although $36 \%$ of drop-outs currently received physiotherapist guidance at baseline when compared with $16 \%$ of completers. Reasons for drop-out were mostly medical or physical complaints (Supplementary Figure 1). The age and work experience of HCPs are presented in Table 2.

\section{Intensive Support Intervention}

Dose Received

Twelve out of 18 trainers $(66.7 \%)$ and all eight dietitians attended the training session before the intervention. At least one physiotherapist and one dietitian of each location attended the training, and trainers who could not attend were informed through E-mail and by their colleagues. Participants attended on average $83.6 \%$ of the delivered training sessions (Table 3). In agreement with the protocol, exercise intensity progressed on average from $47 \%$ of $1 \mathrm{RM}$ in Week $1 \%$ to $75 \%$ of $1 \mathrm{RM}$ in Week 12 . According to the completed registration forms, the intake consultation with the dietitian was received by $98.8 \%$ of intervention participants (average duration $31 \mathrm{~min}$ ), and $91.5 \%$ of participants received the evaluation consultation (average duration $20 \mathrm{~min}$ ). Sixty-six percent of participants used the checklist as a reminder and tool to provide insight into dietary intake.

\section{Acceptability}

Acceptability scores of the intensive support intervention of both participants and HCPs are given in Table 3. Intervention participants and HCPs were highly motivated to start with the intervention. At Week 12, overall satisfaction with the intervention program was rated with a score $8.3 \pm 0.9$ by participants and $7.8 \pm 0.8$ by HCP.

When addressing the exercise component of the intervention, participants and HCP were both satisfied (score $8.3 \pm 1.1$ and $7.3 \pm 0.8$, respectively). Positive points, according to participants, were the group-based program and the physiotherapist guidance. A suggestion for improvement according to both participants and HCPs was increasing variation in exercises besides the resistance exercises.

The diet intervention was also appreciated by participants and HCPs $(7.5 \pm 1.3$ and $7.5 \pm 1.1$, respectively), although on average participants scored the diet 
Table 2. Baseline Characteristics of Intervention Participants, and of Completers and Drop-Outs Within the Intervention Group of the ProMuscle in Practice Intervention

\begin{tabular}{|c|c|c|c|c|}
\hline Characteristics & \multicolumn{2}{|c|}{$\begin{array}{l}\text { Total intervention group } \\
(N=82)\end{array}$} & $\begin{array}{l}\text { Completers } \\
(n=71)\end{array}$ & $\begin{array}{l}\text { Drop-outs before } \\
\text { T2 }(n=11)\end{array}$ \\
\hline Age, mean $\pm S D$ & \multicolumn{2}{|l|}{$74.7 \pm 5.8$} & $74.6 \pm 5.7$ & $75.7 \pm 6.5$ \\
\hline Males, $n(\%)$ & \multicolumn{2}{|l|}{$31(37.8)$} & $29(40.8)$ & $2(18.2)$ \\
\hline \multicolumn{5}{|l|}{ Frailty status, $n(\%)$} \\
\hline Nonfrail & \multicolumn{2}{|l|}{$41(50.0)$} & $36(50.7)$ & $5(45.5)$ \\
\hline Prefrail & \multicolumn{2}{|l|}{$39(47.6)$} & $33(46.5)$ & $6(54.5)$ \\
\hline Frail & \multicolumn{2}{|l|}{$2(2.4)$} & $2(2.8)$ & $0(0.0)$ \\
\hline \multicolumn{5}{|l|}{ Level of education, $n(\%)$} \\
\hline Primary or less & \multicolumn{2}{|l|}{$2(2.4)$} & $1(1.4)$ & $1(9.1)$ \\
\hline Secondary professional or vocational & \multicolumn{2}{|l|}{$54(65.9)$} & $49(69.0)$ & $5(45.5)$ \\
\hline Higher vocational or university & \multicolumn{2}{|l|}{$26(31.7)$} & $21(29.6)$ & $5(45.5)$ \\
\hline \multicolumn{5}{|l|}{ Living situation (together/alone), $n(\%)$} \\
\hline Alone & \multicolumn{2}{|l|}{$32(39.0)$} & $26(36.6)$ & $6(54.5)$ \\
\hline Together & \multicolumn{2}{|l|}{$50(61.0)$} & $45(63.4)$ & $5(45.5)$ \\
\hline Care use, $n(\%)$ & \multicolumn{2}{|l|}{$11(13.4)$} & $8(11.3)$ & $3(27.3)$ \\
\hline \multicolumn{5}{|l|}{ Morbidities, $n(\%)$} \\
\hline Diabetes $^{\mathrm{a}}$ & \multicolumn{2}{|l|}{$9(11.0)$} & $7(9.9)$ & $2(18.2)$ \\
\hline Arthrosis & \multicolumn{2}{|l|}{$38(46.3)$} & $32(45.1)$ & $6(54.5)$ \\
\hline Fracture & \multicolumn{2}{|l|}{$3(3.7)$} & $2(2.8)$ & $1(9.1)$ \\
\hline Other & \multicolumn{2}{|l|}{$69(84.1)$} & $61(85.9)$ & $8(72.7)$ \\
\hline Current physiotherapist guidance (yes), $n(\%)$ & \multicolumn{2}{|l|}{$15(18.3)$} & $11(15.5)$ & $4(36.4)$ \\
\hline Currently on a diet (yes), $n(\%)$ & \multicolumn{2}{|l|}{$10(12.2)$} & $9(12.7)$ & $1(9.1)$ \\
\hline \multirow[t]{3}{*}{ History of sports (yes), $n(\%)$} & \multicolumn{2}{|l|}{$70(85.4)$} & $60(84.5)$ & $10(90.9)$ \\
\hline & \multicolumn{2}{|c|}{ Intensive support intervention } & \multicolumn{2}{|c|}{ Moderate support intervention } \\
\hline & $\begin{array}{l}\text { Physiotherapists } \\
(n=18)\end{array}$ & $\begin{array}{l}\text { Dietitians } \\
(n=8)\end{array}$ & $\begin{array}{l}\text { Exercise trainers } \\
(n=9)\end{array}$ & $\begin{array}{l}\text { Nutrition course leaders } \\
(n=2)^{\mathrm{b}}\end{array}$ \\
\hline Age, mean $\pm S D$ (range) & $\begin{array}{l}29.7 \pm 12.2 \\
(18-58)\end{array}$ & $\begin{array}{l}33.9 \pm 13.5 \\
(22-64)\end{array}$ & $\begin{array}{l}46.4 \pm 15.4 \\
(21-62)\end{array}$ & $\begin{array}{l}25.5 \pm 3.5 \\
(23 \text { and } 28)\end{array}$ \\
\hline Work experience in years, mean $\pm S D$ (range) & $\begin{array}{l}7 \pm 1.03 \\
(0-37)\end{array}$ & $\begin{array}{l}10.1 \pm 13.7 \\
(0-41)\end{array}$ & $\begin{array}{l}18.6 \pm 14.8 \\
(3-40)\end{array}$ & $\begin{array}{l}4 \pm 4.2 \\
(1 \text { and } 7)\end{array}$ \\
\hline
\end{tabular}

${ }^{\mathrm{a}}$ Data available for $n=81$.

${ }^{b}$ One course leader implemented the nutrition course in the first two municipalities, the other in the last three municipalities.

intervention lower than the exercise intervention. Positive aspects were the consultations with the dietitian and the fact that the dietitian took participants' dietary preferences into account in the advice. Both participants and HCPs indicated that the number of protein-rich foods added to the diet was high, and that it would be better to include more variation.

Qualitative data showed that HCPs were satisfied with the training session that they received beforehand and with the clear manuals and registration forms, which contained enough information to be able to start with the intervention. In general, HCPs felt sufficiently involved in the intervention. Overall, HCPs were satisfied with the multidisciplinary collaboration. They perceived the joint peer discussion in Week 6 as useful, as it helped to exchange experiences with the other professionals and to discuss bottlenecks with regard to implementation (e.g., the situation of certain participants, communication between professionals, logistic issues with distributing the proteinrich foods). However, in several locations, physiotherapists indicated that they would have preferred more contact with the dietitians concerning diet-related questions from participants.

\section{Fidelity and Applicability}

Resistance exercise.-Overall, the exercise intervention was implemented as planned, with physiotherapists following the progressive training protocol (Table 3) and supporting and motivating participants. Group size varied between four and seven participants, and there were two or three trainers per group. Physiotherapists indicated in the interviews that group coherence was achieved automatically, but that they also facilitated this by starting 
Table 3. Dose Received and Acceptability of the Intensive Support Intervention for Participants and Health Care Professionals

\begin{tabular}{|c|c|c|}
\hline & $\begin{array}{l}\text { Dose according to } \\
\text { protocol }\end{array}$ & $\begin{array}{l}\text { Received by } \\
\text { participants }\end{array}$ \\
\hline \multicolumn{3}{|l|}{ Dose received } \\
\hline \multicolumn{3}{|l|}{ Resistance exercise sessions } \\
\hline Number of exercise sessions attended, $n(\%)^{\text {a }}$ & 24 sessions & $19.7(83.6)$ \\
\hline \multicolumn{3}{|l|}{ Mean (Weeks 1-12) intensity of the exercises (\% of $1 \mathrm{RM}$ ) } \\
\hline Leg press & Not specified & 63 \\
\hline Leg extension & Not specified & 62 \\
\hline Mean intensity at Week $1(\% \text { of } 1 \mathrm{RM} \text { at Week } 1)^{\mathrm{b}}$ & $50 \%$ of $1 \mathrm{RM}$ & 47 \\
\hline Mean intensity at Week $5(\% \text { of } 1 \mathrm{RM} \text { at Week } 1)^{\mathrm{b}}$ & $60 \%-75 \%$ of $1 \mathrm{RM}$ & 66 \\
\hline Mean intensity at Week $12(\% \text { of } 1 \mathrm{RM} \text { at Week } 6)^{\mathrm{b}}$ & $70 \%-80 \%$ of $1 \mathrm{RM}$ & 75 \\
\hline \multicolumn{3}{|l|}{ Mean (Weeks 1-12) number of sets per exercise (mean) ${ }^{c}$} \\
\hline Leg press & 4 sets & 3.5 \\
\hline Leg extension & 4 sets & 3.5 \\
\hline \multicolumn{3}{|l|}{ Mean (Weeks 1-12) number of repetitions per set } \\
\hline Leg press & Varies over time & 12.4 \\
\hline Leg extension & (range: $8-15$ repetitions) & 12.3 \\
\hline \multicolumn{3}{|l|}{ Diet intervention } \\
\hline Number of participants who received intake consultation, $n(\%)^{\mathrm{d}}$ & $100 \%$ & $81(98.8)$ \\
\hline Intake consultation performed before Week $1, n(\%)^{\mathrm{d}}$ & $100 \%$ & $35(42.7)$ \\
\hline Number of participants who received evaluation consultation, $n(\%)^{\mathrm{d}}$ & $100 \%$ & $75(91.5)$ \\
\hline Evaluation consultation performed in Weeks 5,6 , or $7, n(\%)^{\mathrm{d}}$ & $100 \%$ & $67(81.7)$ \\
\hline Dietary advice adjusted during evaluation consultation, $n(\%)^{\mathrm{d}}$ & Optional & $30(36.6)$ \\
\hline $\begin{array}{l}\text { Dietitians informed participants about moderate support intervention during } \\
\text { evaluation consultation, } n(\%)^{\mathrm{d}}\end{array}$ & $100 \%$ & $62(75.6)$ \\
\hline \multirow[t]{2}{*}{ Number of participants who received an additional consultation, $n(\%)$} & Optional & $9(11.0)$ \\
\hline & Participants & $\begin{array}{l}\text { Health care } \\
\text { professionals }\end{array}$ \\
\hline \multicolumn{3}{|l|}{ Motivation to start intensive support intervention ${ }^{\mathrm{e}}$} \\
\hline Exercise sessions, mean $\pm S D$ & $4.6 \pm 0.5$ & $8.3 \pm 0.8$ \\
\hline Diet intervention, mean $\pm S D$ & $4.4 \pm 0.7$ & $8.3 \pm 0.3$ \\
\hline \multicolumn{3}{|l|}{ Acceptability ${ }^{\mathrm{f}}$} \\
\hline Overall score (scale $1-10)$, mean $\pm S D$ & $8.3 \pm 0.9$ & $7.8 \pm 0.8$ \\
\hline Resistance exercise sessions (scale $1-10$ ), mean $\pm S D$ & $8.3 \pm 1.1$ & $7.3 \pm 0.8$ \\
\hline \multicolumn{3}{|l|}{ Satisfaction with ... (scale $1-5)$, mean $\pm S D$} \\
\hline Physiotherapist explanation of the exercises and training program & $4.7 \pm 0.5$ & N/A \\
\hline Guidance by the physiotherapist during the training sessions & $4.7 \pm 0.6$ & N/A \\
\hline The exercises & $4.6 \pm 0.6$ & N/A \\
\hline Exercising in a group & $4.8 \pm 0.4$ & N/A \\
\hline Extent to which they were being informed of personal training progress & $4.2 \pm 1.0$ & N/A \\
\hline Extent to which they were being informed about the moderate support intervention & $3.7 \pm 1.0$ & N/A \\
\hline Diet intervention (scale 1-10), mean $\pm S D$ & $7.5 \pm 1.3$ & $7.5 \pm 1.1$ \\
\hline \multicolumn{3}{|l|}{ Satisfaction with ... (scale 1-5), mean $\pm S D$} \\
\hline Intake consultation with the dietitian & $4.1 \pm 0.9$ & N/A \\
\hline Evaluation consultation with the dietitian & $4.1 \pm 0.9$ & N/A \\
\hline Number of protein-rich foods to consume daily & $3.8 \pm 1.1$ & N/A \\
\hline Filling out the checklist every day & $3.7 \pm 1.1$ & N/A \\
\hline Extent to which they were being informed about the moderate support intervention & $3.6 \pm 1.0$ & N/A \\
\hline
\end{tabular}

Note: $1 \mathrm{RM}=1$ repetition maximum.

${ }^{a}$ In Apeldoorn, Epe, Ermelo, and Harderwijk, 24 training sessions were offered; in Ede, 22 training sessions were offered.

${ }^{\mathrm{b}}$ Combined for leg press and leg extension machine.

'Excluding data from Ede.

${ }^{\mathrm{d}}$ Based on available data from the registration forms (data available for $71-81$ participants, depending on item). Percentage presented in Table is based on all 82 intervention participants.

'Scale 1-5 for participants, scale 1-10 for health care professionals.

'Participants $n=74$, professionals: overall acceptability $n=26$, resistance exercise acceptability $n=18$ physiotherapists, diet intervention acceptability $n=8$ dietitians. 
conversations within the group, or by performing a groupbased warm-up or warm-down.

A deviation from the manual was that physiotherapists did not map the abilities and constraints of participants at the beginning of the intervention program, although in their daily work, they would do so. They indicated that they received information about participants' strength and medical information too late from the researchers, which hindered tailoring the training intensity at the start of the training program. Physiotherapists deviated from the training protocol when necessary, that is, in case of physical complaints. In the last weeks of the intervention period, physiotherapists reported less training intensity progression because participants had reached their maximum. In some locations, other or adjusted resistance exercises were performed for part of the intervention period, as some of the training machines did not function properly. At all locations, an intermediary strength test was performed around Week 6, used to evaluate training progression and inform participants about their progress.

Dietary protein intervention.-On the basis of the completed registration forms, $42.7 \%$ of the intake consultations were performed before the start of the intervention period, following the manual (Table 3). All dietitians formulated the dietary advice together with the participant, and dietitians spoke about most of the required topics during the intake. The contact moment in the first training week was not performed as planned, as this was done mostly in later weeks according to the registration forms. Participants addressed questions about the dietary intervention mostly to the physiotherapists in the first weeks. Dietitians performed evaluation consultations around Week 6 with the majority of participants $(81.7 \%)$, upon which dietary advice was adjusted for $37 \%$ of participants, and $76 \%$ of participants were informed about the moderate support intervention. Due to logistic issues with protein-rich foods, participants did not always receive the advised foods.

Fit with target group and with HCP working procedures.HCPs expressed in the interviews that the intervention fitted well with the target population. They mentioned that participants noticed positive intervention effects, especially in ADL functioning and perceived fitness (e.g., climbing the stairs was easier, less tired after walking). HCPs indicated that the intervention fitted their regular working procedures, and dietitians and physiotherapists have the required competencies, including people skills and knowledge. The involved HCP worked mostly with a more frail population in long-term care or rehabilitation and indicated that with the current target group this intervention would fit best in primary care. The involved HCP would like to continue working with the intervention, with preconditions being sufficient time available to implement the project, and recruiting the community-dwelling target population.

\section{Moderate Support Intervention}

Table 4 and Supplementary Table 1 show an overview of the moderate support intervention components offered per municipality. Four municipalities offered sessions in a fitness center, one municipality in a sports hall and three municipalities with a primary-care physiotherapist. The type of exercises performed and costs for participants varied between municipalities. The nutrition course had the same general format at all municipalities.

\section{Transfer to Moderate Support Intervention}

Participants were informed on the moderate support intervention approximately 4-5 weeks before the start through a letter and a leaflet, except in Apeldoorn where the program was confirmed at a late stage, and participants were informed only 2 weeks before the start. Participants were relatively satisfied with the information they received about the moderate support intervention from physiotherapists and dietitians $(3.7 \pm 1.0$ and $3.6 \pm 1.0$, respectively, on a 5 -point scale, Table 3) and were motivated to participate. HCPs indicated that they were not informed on the exact moderate support intervention content in a timely manner, so they had difficulty properly informing the participants about the program. However, HCPs were satisfied with the moderate support intervention content as it includes the combination of diet and exercise, sufficient guidance, and low costs.

\section{Dose Received}

Some exercise providers offered two sessions a week, while others offered one session a week (Table 4). Each exercise option was attended by $4-8$ participants per municipality $(56.1 \%$ of all intervention participants attended one or more exercise sessions), who attended between $43.8 \%$ and $83.3 \%$ of delivered sessions (mean $63.6 \%$ ) (Table 4 ; Supplementary Table 1). The main reasons for joining the exercise sessions were health, practical location or time (in the morning), and social aspects (social interaction or continuing together with their intensive support intervention training group). The nutrition course was attended by 3-13 participants per municipality $(59.8 \%$ of participants in total), who attended on average $76.8 \%$ of the course sessions (data of Epe is missing; Table 5). During the intervention period, the majority of participants indicated to have tried to consume $25 \mathrm{~g}$ of protein during breakfast $(n=54)$ or lunch $(n=50)$. The newsletter was read by 58 participants, and the majority found the newsletter useful. The main reasons for nonparticipation in the moderate support intervention were inconvenient planning, having no interest in nutrition, having physical complaints, prefer exercising outside, and because not all intensive support intervention group members continued.

\section{Acceptability}

Sixty-six participants completed the questionnaire after the moderate support intervention. Participants' average 


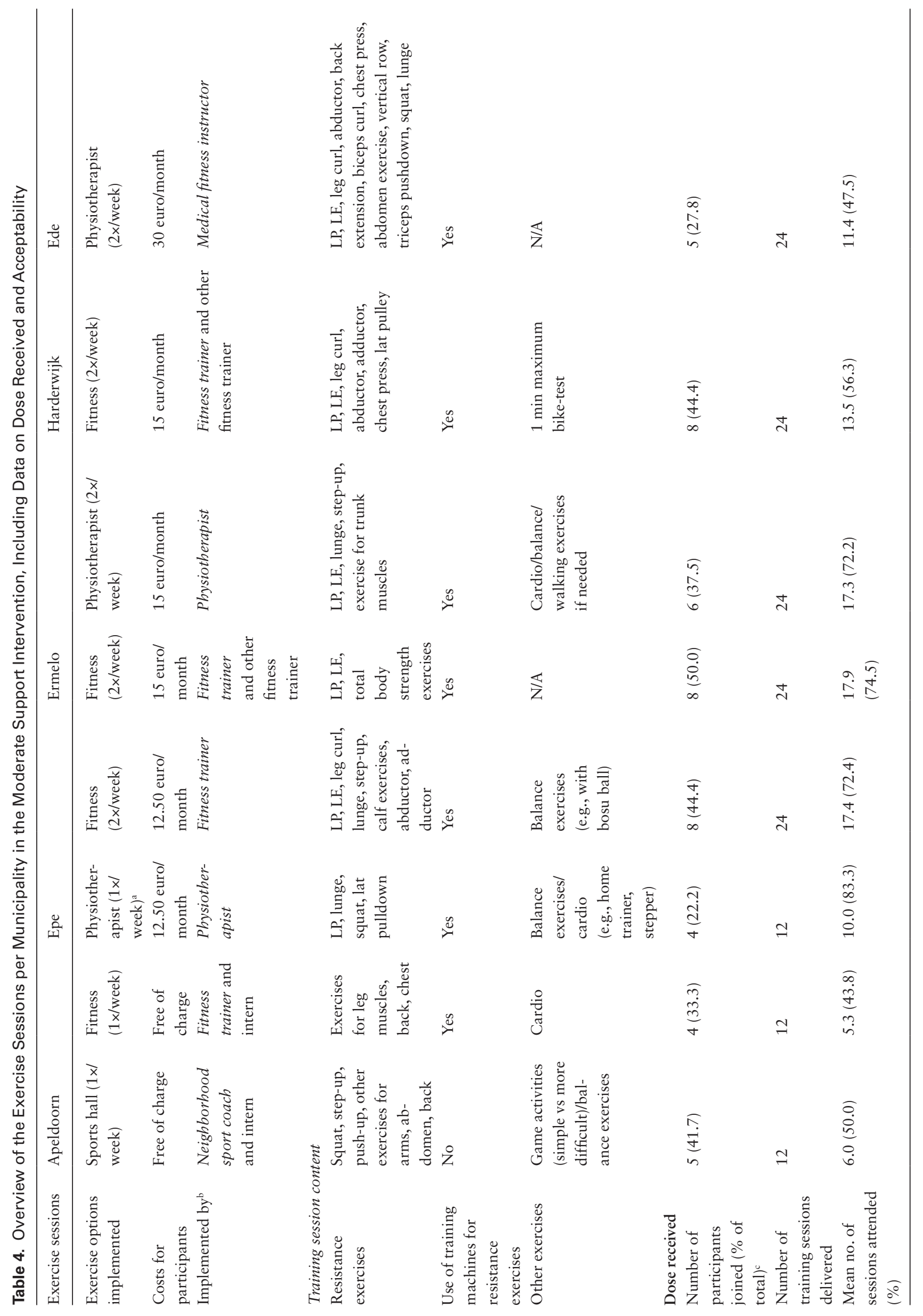




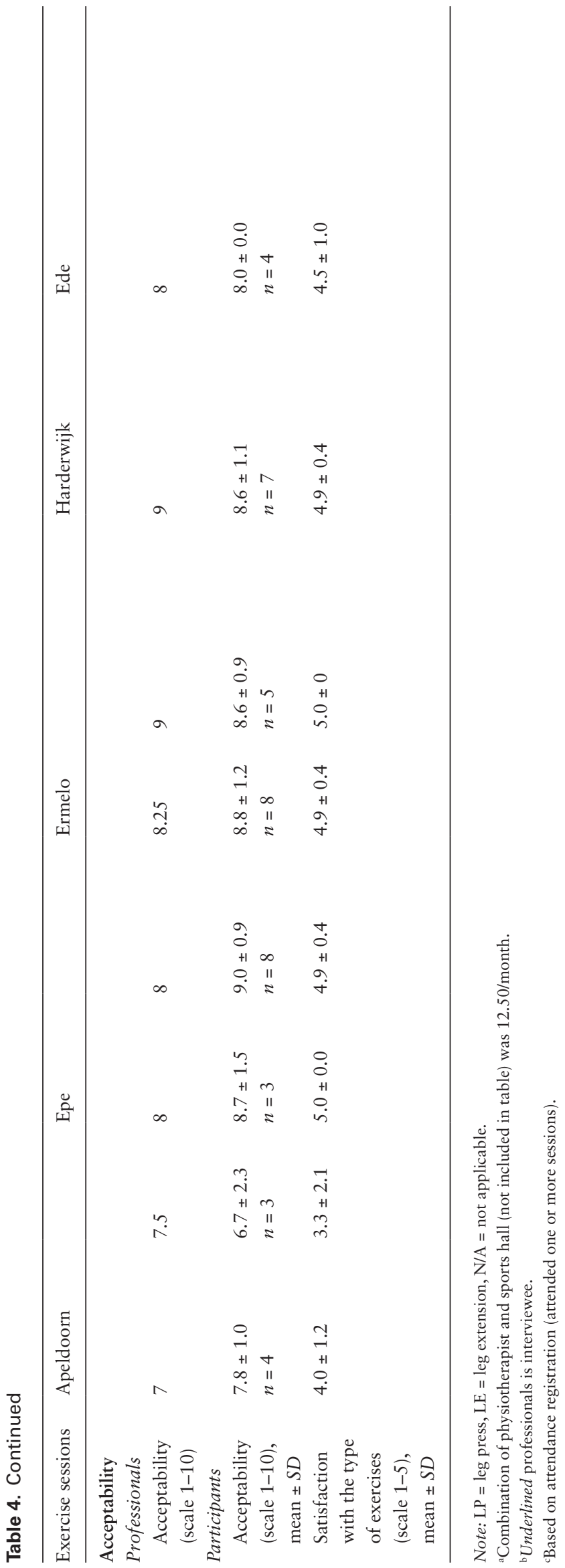

acceptability of the total moderate support intervention was $8.1 \pm 1.3(n=58)$. The majority of participants could fit the moderate support intervention well within their daily life $(n=52)$ and perceived it to be of added value $(n=49)$. Both nutrition course leaders and all but one exercise trainer were motivated to implement the moderate support intervention (Supplementary Table 1 and Table 5) and felt generally involved with the project. However, both nutrition course leaders indicated that they missed a connection with the overall project and the exercise trainers, and lacked insight in the diet intervention of the first 12 weeks.

Exercise sessions.-On average, participants and exercise trainers were satisfied with the exercise sessions, mean scores $8.4 \pm 1.2$ and $8.1 \pm 0.7$, respectively. Participants were satisfied overall with the type of exercises, supervision, and group-based training (Table 6). When comparing the types of exercise options, both participant and trainer satisfaction with exercise in a sports hall was lower than satisfaction with fitness centers and with primary-care physiotherapists (Table 6), and the exercise sessions in the sports hall matched somewhat less with exercises from the intensive support intervention (Supplementary Table 1).

Similarly to the intensive support intervention, trainers considered it positive that the project made older adults aware of the importance of both resistance exercise and nutrition, that the trainings were in groups, and that they saw improvements in ADL, balance, and exercise intensity. After the study, some involved fitness centers and primary-care physiotherapists continued the training group and indicated that several participants became a member of their organization. However, they mentioned that, for other participants, membership costs were a barrier for continuation.

Nutrition course.-Participants were satisfied with the group-based nutrition course (mean score $8.1 \pm 0.8$ ), the information, and the activities during the course (Tables 5 and 6). In the questionnaire and interviews, participants indicated that they learned new things about protein-rich foods and meals, became more aware of the importance of a protein-rich diet, and obtained more insight into their protein intake. A point of improvement could be the addition of more general dietary advice instead of focusing mostly on proteins and more personal dietary advice.

The nutrition course leaders were moderately satisfied with the nutrition course (mean score $6.8 \pm 0.8$ ), with the lowest satisfaction for the first course in Apeldoorn, as the leader experienced it as a sort of try-out. The strong points were the practical aspects of the course and the fact that it was organized in the local setting of participants.

Fidelity and Applicability

Exercise intervention.-Exercise providers used the manuals as inspiration and based the training session content on possibilities within their setting and 


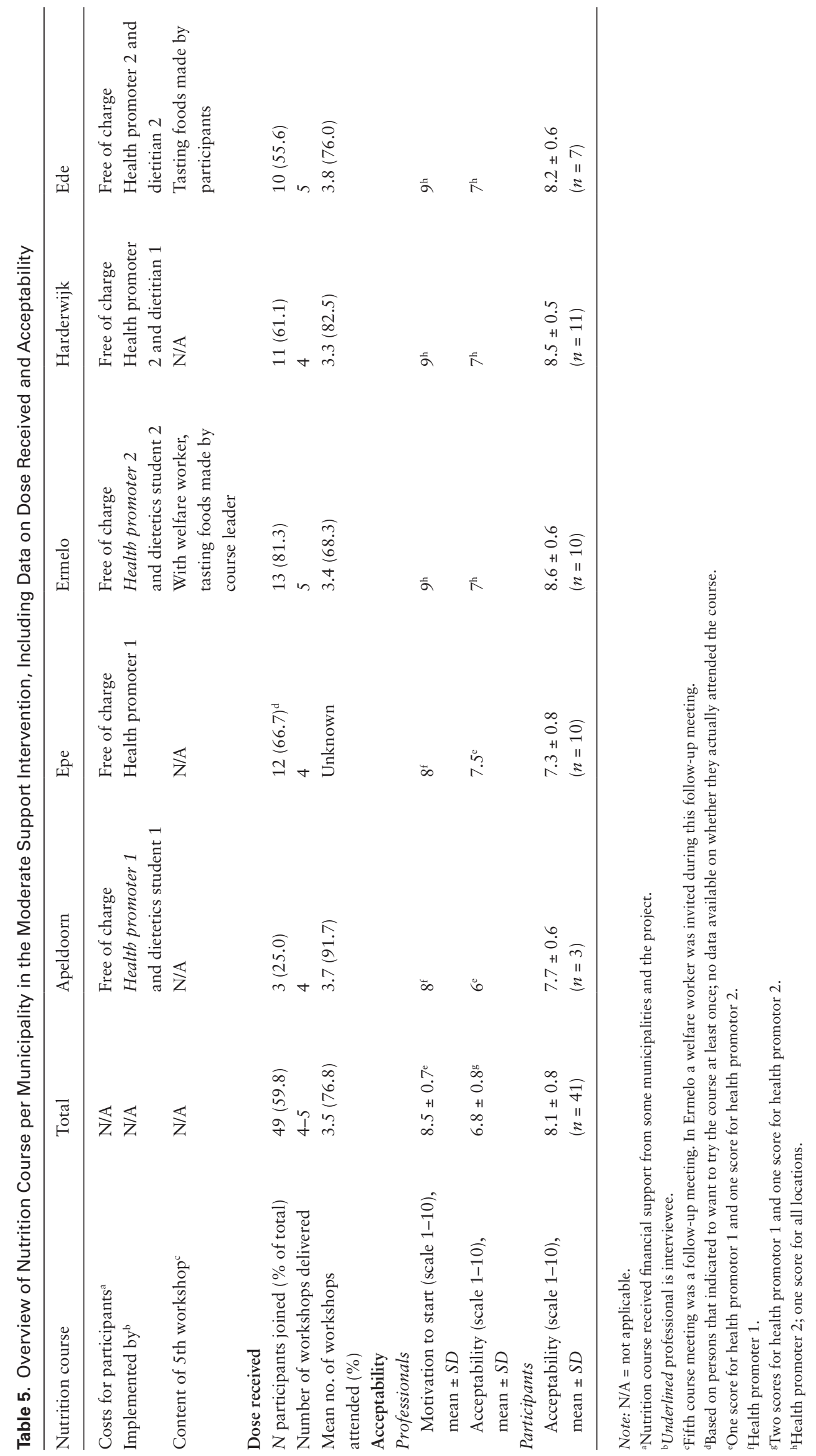


participants' abilities. In the first location, Apeldoorn, the manual was still in development, so the trainers did not have access to a list with exemplar resistance exercises. The majority of trainers added other types of exercise to sessions, such as balance or functional exercises, cardio, exercises in circuit form, or sport games (Table 4). All trainers except the fitness trainer in Apeldoorn indicated to have increased training intensity, although some trainers indicated that it was difficult to keep track of the intensity level. In general, the trainer focused solely on the training group, while the fitness trainer in Apeldoorn was supervising the whole fitness center.

In larger groups, trainers struggled to divide attention over the participants with explaining the exercises in the beginning. During the sessions, trainers explained exercises and paid attention to correct performance of the exercises. To motivate participants, trainers tried to make participants see their training success and stimulated them to increase exercise intensity. Several trainers tried to facilitate group cohesion, mainly by including group-based exercises in circuit form, or by facilitating a coffee moment after the training (Supplementary Table 1). In the sports hall in Apeldoorn, additional materials (e.g., free weights) would have facilitated the performance of more resistance exercises.

Nutrition course.-Two course leaders were consecutively involved in the nutrition course, and they received assistance from a dietitian or dietetics student during course sessions (Table 5). The first course leader developed the course manual, and the second course leader adjusted among other things the recipes and developed slideshows. From the third location onward, the participants received the slideshows, materials, and recipes after the course.

Qualitative data showed that 1.5 -hr workshops were perceived as rather short by the course leaders, especially when cooking the recipes. During the sessions, the leaders started with a recap of the previous session and discussed theory focused mostly on dietary proteins. Course leaders indicated in the interview that social interaction was very important, and that participants generally were eager to give examples and to discuss their dietary habits. Course leaders also indicated that the fifth workshop was canceled in three municipalities as participants were not interested in a supermarket visit and was held in Ermelo and Ede several weeks after meeting four the fourth workshop.

Fit with target group and HCP working procedures.According to HCPs, both the exercise sessions and the nutrition course were suitable for the target group. In the questionnaire, all except three participants indicated that the exercise intensity was just right, and not too light or heavy. Participants noted a difference in the information they received from the dietitian in the intensive support intervention (mainly dairy-focused) and the information as included in the nutrition course (i.e., broader information on protein, reading labels).

HCPs indicated that the intervention fitted with their working procedures. All trainers except the neighborhood sports coach in Apeldoorn were used to supervising groupbased training and working with this target group. Some mentioned differences from their normal work, that is, that they would usually perform an individual intake with participants or would use a different build-up of sets and repetitions. Trainers indicated to miss information on possible physical complaints or medical issues of participants. Nutrition course leaders were also used to work in health promotion and education.

\section{Discussion and Implications}

The aim of this comprehensive process evaluation was to obtain insight into implementation and context of the ProMuscle in Practice resistance exercise and dietary protein intervention. The intensive support intervention was implemented with high dose received and fidelity, and was accepted by both participants and implementing HCPs. The moderate support intervention had lower attendance rates, and especially the exercise session content and acceptability showed high variability across settings.

On the basis of this process evaluation, we can suggest key elements of the intervention and implementation that explain how the intervention produces change, also known as the mechanisms of impact (Moore et al., 2015). We observed high dose received for the intensive support intervention, comparable with the original clinical study (Tieland et al., 2012), and lower and more variable dose received for the moderate support intervention. The lower dose received in the optional moderate support intervention could indicate that participants felt less obliged to join all sessions, or that intervention content did not always match with participants' needs. We observed that not all participants continued with the moderate support intervention, suggesting that the transfer to this intervention period was not optimal. One possible explanation for this is that HCPs from the first period were informed too late about the moderate support intervention content, and were, therefore, not able to sufficiently motivate participants to continue. Lack of information is a barrier to participating in physical activity programs (Bethancourt, Rosenberg, Beatty, \& Arterburn, 2014), emphasizing the need for sufficient information supply about the follow-up program. Practical reasons for noncontinuation with the moderate support intervention, such as inconvenient location or lack of time, are in line with other research (Olanrewaju, Kelly, Cowan, Brayne, \& Lafortune, 2016). However, as increased contact frequency within combined lifestyle interventions is associated with increased intervention effectiveness (Greaves et al., 2011), increasing adherence over time is important. For future intervention continuation, more attention should be paid to a better transfer to 
community facilities and overcoming barriers experienced by participants for this transfer.

Intervention acceptability may be a prerequisite to achieve high dose received. Overall, participants were satisfied with both intervention periods. Participants appreciated the group-based nature of the intervention, even though they did not indicate the social aspect to be an important reason to participate at baseline. Although evidence of the impact of social support on intervention effectiveness is conflicting (Greaves et al., 2011; Zubala et al., 2017), studies investigating motivators and preferences of older adults for participating in physical activity or nutritional programs identified fun and social interaction as important factors (Dedeyne et al., 2018; DevereuxFitzgerald, Powell, Dewhurst, \& French, 2016; Olanrewaju et al., 2016). Especially the presence of like-minded peers seems to be beneficial (Hardy \& Grogan, 2009), and these findings support the importance of social aspects within the intervention. In our study, it appeared that there was most room for social interaction in the moderate support intervention, for example, during circuit-form training and during nutrition course activities. HCPs have an important role in facilitating social cohesion and stimulating communication to create social support. Furthermore, promoting continuation of the intervention with the same group may be helpful to achieve long-term behavior change.
Other intervention elements that contributed to intervention acceptability for participants were tailoring the intervention and intensive HCP supervision. Most tailoring occurred within the intensive support intervention as this included more intensive training guidance and individual dietary advice. A person-centered approach was identified as an important element for physical activity intervention effectiveness for older adults (Olanrewaju et al., 2016; Zubala et al., 2017). However, at the start of both the intensive support and moderate support intervention, HCPs often could not tailor the program to participants' capabilities or needs as they lacked information on medical issues of participants. Therefore, HCPs from both intervention periods would prefer to perform an intake at the start, as they would normally. Performing a multidisciplinary intake beforehand could possibly prevent drop-out, and elicit input for specific types of exercise (e.g., balance, functional) that could help participants to achieve their goals. On the basis of our results, intensive professional supervision is needed to tailor the intervention and to apply the other behavior change techniques (e.g., feedback, goal setting) that are necessary to change participants' behavior (van Dongen et al., 2018). Goal setting (Cullen, Baranowski, \& Smith, 2001; Olanrewaju et al., 2016) and feedback (O'Brien et al., 2015) are examples of behavior change techniques that can positively impact intervention effectiveness.

Table 6. Participants' Acceptability of the Moderate Support Intervention Components, for the Exercise Sessions Also Clustered for Sports Hall, Fitness, or Physiotherapist

\begin{tabular}{|c|c|c|c|c|}
\hline Exercise sessions & $\begin{array}{l}\text { Total } \\
(N=43)^{a}\end{array}$ & $\begin{array}{l}\text { Sports hall } \\
(n=5)\end{array}$ & $\begin{array}{l}\text { Fitness } \\
(n=27)\end{array}$ & $\begin{array}{l}\text { Physiotherapist } \\
(n=12)\end{array}$ \\
\hline Participants' motivation to start (scale $1-5)($ scale $1-5)$, mean $\pm S D^{a}$ & $3.9 \pm 1.0$ & N/A & N/A & N/A \\
\hline Participants' acceptability (scale $1-10)$ (scale $1-5)$, mean \pm SD & $8.4 \pm 1.2$ & $7.8 \pm 1.0$ & $8.5 \pm 1.4$ & $8.4 \pm 0.9$ \\
\hline \multicolumn{5}{|l|}{ Satisfaction with ... (scale $1-5)$} \\
\hline Type of exercise & $4.7 \pm 0.8$ & $4.0 \pm 1.2$ & $4.7 \pm 0.8$ & $4.8 \pm 0.6$ \\
\hline Supervision during training sessions & $4.9 \pm 0.3$ & $5.0 \pm 0.0$ & $4.8 \pm 0.4$ & $5.0 \pm 0.0$ \\
\hline Group-based training & $4.8 \pm 0.4$ & $4.8 \pm 0.4$ & $4.8 \pm 0.4$ & $4.8 \pm 0.4$ \\
\hline Nutrition course & & $\begin{array}{l}\text { Total } \\
(N=41)\end{array}$ & & \\
\hline Participants' motivation to start (scale $1-5)$, mean $\pm S D$ & & $4.0 \pm 0.8$ & & \\
\hline Participants' acceptability (scale $1-5$ ), mean $\pm S D$ & & $8.1 \pm 0.8$ & & \\
\hline \multicolumn{5}{|l|}{ Because of participating in the nutrition course ... (scale $1-5)^{\mathrm{b}}$} \\
\hline I gained more insight in personal protein intake & & $4.1 \pm 1.2$ & & \\
\hline Gained ideas on protein-rich meals & & $4.1 \pm 1.0$ & & \\
\hline Learned new things about protein-rich nutrition & & $4.1 \pm 1.1$ & & \\
\hline Know how to use the info in daily life & & $4.1 \pm 1.0$ & & \\
\hline \multicolumn{5}{|l|}{ Satisfaction with ... (scale $1-5$ ) } \\
\hline Information received during the dietary workshops & & $4.8 \pm 0.5$ & & \\
\hline Group-based dietary workshops & & $4.9 \pm 0.3$ & & \\
\hline The preparation of protein-rich breakfast meals? & & $4.7 \pm 0.5$ & & \\
\hline The preparation of protein-rich lunch meals? & & $4.7 \pm 0.6$ & & \\
\hline The preparation of protein-rich dinner meals? & & $4.7 \pm 0.5$ & & \\
\hline Viewing product labels & & $4.7 \pm 0.6$ & & \\
\hline
\end{tabular}

${ }^{a}$ One participant joined both the sport center and fitness, so is included once in the total score.

${ }^{\mathrm{b}} \mathrm{S}$ core 1 (totally disagree) to 5 (totally agree). 
A combination of education and behavioral activities may work best for older adults to improve physical activity behavior (Chase, 2015). Although face-to-face contact with a professional is not necessary for physical activity intervention effectiveness (Chase, 2015; Olanrewaju et al., 2016), it can increase the effect size (Olanrewaju et al., 2016), with more intensive contact with HCPs resulting in higher effect sizes (Conn, Valentine, \& Cooper, 2002). We therefore consider professional supervision essential for our target group and contributing to intervention acceptability.

The diet intervention in the intensive support intervention included a personal, tailored approach including facilitation with protein-rich foods, whereas the moderate support intervention used a less-individual approach. We saw the largest increases in dietary protein intake in the first period, with only slight decreases in protein intake in the second 12 weeks (van Dongen et al., 2020). This indicates that participants were reasonably able to maintain their increased protein intakes after cessation of receiving protein-rich foods, although on average reaching the $25 \mathrm{~g}$ threshold over time seems to be most difficult for the breakfast meal. Qualitative research shows that fit of dietary protein foods with older adults' habits and health benefit knowledge are important drivers for consuming protein-rich foods (Herrema, Westerman, van Dongen, Kudla, \& Veltkamp, 2018), which are aspects incorporated in the intervention. However, food choice in older adults is a complex interplay between numerous factors (Host, McMahon, Walton, \& Charlton, 2016), and thus sufficient tailoring of dietary advice is needed to improve dietary patterns. Tailoring of dietary advice was incorporated in the intensive support period, but enabling the dietitian to be present more often during the training sessions to answer questions may be helpful, as the checklists proved to be unsuccessful. Overall, the largest intervention effects and acceptability were seen in the intensive support intervention (van Dongen et al., 2020), which might indicate that a more tailored and intensively supervised intervention is most effective in improving health outcomes and achieving participant satisfaction.

This multicenter design allowed us to get insight into intervention fit and adaptations to context in several locations. In general, HCPs indicated the intervention to fit their working procedures, an important prerequisite to achieve implementation success. Implementation fidelity differed between the intensive support intervention and the moderate support intervention, mostly for the exercise sessions. Physiotherapists generally adhered to the implementation manuals in the first 12 weeks, whereas we observed more variation in training content in the second 12 weeks. The latter intervention was not previously tested, and the manuals were not very strict, so we expected more implementation variation. In the moderate support intervention, the main focus was still on resistance training, but sessions also included other types of exercises. This matched with wishes from participants who missed variation in exercises during the intensive support intervention. Adapting or selecting only parts of an intervention might support intervention sustainability in daily practice, as the implementers can fit the intervention to the needs of themselves and the participants (Craig et al., 2013; Petrescu-Prahova, Belza, Kohn, \& Miyawaki, 2016). Overall, the moderate support exercise intervention fitted best within fitness centers or primary-care physiotherapy practices. On the basis of our results, we propose that the moderate support intervention exercise sessions should include skilled supervisors that monitor progress and provide feedback, training in groups, and a combination of resistance exercise on training machines and additional exercises.

Besides implementation fidelity and fit in the real-life setting, multidisciplinary collaboration also contributes to implementation success. The physiotherapists and dietitians, in general, had a good contact in the intensive support intervention, as they worked in the same organization and appreciated the joint peer discussion. However, cohesion between the exercise and nutrition component of the moderate support intervention, and connection with the first intervention period, was lacking. Targeting both exercise and nutrition in this intervention is a strength (Greaves et al., 2011), which was also confirmed by the HCPs. Further intertwining the exercise and nutrition aspects in both intervention periods, and strengthening the collaboration between all involved organizations, may make the intervention more coherent.

Some strengths and limitations related to the study design should be mentioned. We believe that the high fidelity to content and overall high acceptability by HCPs and participants was due to the systematic intervention adaptation and piloting process (van Dongen et al., 2017). A strength of this study was the comprehensive process evaluation that used suitable frameworks to guide evaluation efforts. Secondly, the involved organizations could be viewed as early adopters (Rogers, 2003) and might not, therefore, fully represent other health care organizations in the Netherlands. Nevertheless, multiple practice organizations were involved in the different municipalities, proving that it is possible to successfully implement the intervention in practice. Currently, the intensive support intervention was implemented in secondary care, while based on the target population and the professionals, this intervention would fit better within primary care or within public health. Additionally, as the intervention had a phased start in the different municipalities, the intervention, materials, and recruitment procedures were continuously improved during the study. We expect that the later intervention locations benefitted from this ongoing improvement in, for example, the number of recruited participants and the organization of the moderate support intervention.

In conclusion, the ProMuscle in Practice intervention was feasible to implement and generally acceptable to both community-dwelling older adults and implementing HCPs. 
The intensive support intervention was implemented with high dose received and fidelity, whereas for the moderate support intervention, the dose received and implementation were more variable between settings. Key elements that we assume contribute to intervention success were tailored interventions, intensive supervision by skilled health care professionals, social aspects, implementation fidelity, the fit within the real-world setting, and multidisciplinary collaboration. The moderate support intervention should receive due attention in future implementation to achieve optimal participant engagement and intervention delivery including key intervention elements. Continuous intervention optimization, while taking into account the key intervention elements, is warranted for broader implementation of this combined intervention for community-dwelling older adults.

\section{Supplementary Material}

Supplementary data are available at The Gerontologist online.

\section{Funding}

This work was supported by financial support from the Dutch Ministerie van Economische Zaken, FrieslandCampina, and Innopastry [TKI-AF-15206]. Neither organization had any role in the design, analyses, or writing of this article.

\section{Acknowledgments}

We thank all participants, health care professionals, care organizations (Zorggroep Apeldoorn en omstreken, Viattence, Zorggroep Noordwest-Veluwe, Opella), local organizations, municipalities, students, and research assistants involved in the ProMuscle in Practice study, with special thanks to Nick Wezenbeek and Lilian Brouwer. The ProMuscle in Practice project is a public-private partnership. The public partners are responsible for the study design, data collection and analysis, decision to publish, and preparation of the article. The private partners (FrieslandCampina, Innopastry, Nutrition and Healthcare Alliance, Zilveren Kruis) have contributed to the project through regular discussion, and financial and in-kind contributions.

\section{Conflict of Interest}

None reported.

\section{References}

Bethancourt, H. J., Rosenberg, D. E., Beatty, T., \& Arterburn, D. E. (2014). Barriers to and facilitators of physical activity program use among older adults. Clinical Medicine \& Research, 12, 10 20. doi: $10.3121 / \mathrm{cmr} .2013 .1171$

Cermak, N. M., Res, P. T., de Groot, L. C., Saris, W. H., \& van Loon, L. J. (2012). Protein supplementation augments the adaptive response of skeletal muscle to resistance-type exercise training: A meta-analysis. The American Journal of Clinical Nutrition, 96, 1454-1464. doi:10.3945/ajcn.112.037556

Chase, J.-A. D. (2015). Interventions to increase physical activity among older adults: A meta-analysis. The Gerontologist, 55(4), 706-718. doi:10.1093/geront/gnu090

Conn, V. S., Valentine, J. C., \& Cooper, H. M. (2002). Interventions to increase physical activity among aging adults: A meta-analysis. Annals of Behavioral Medicine, 24, 190-200. doi:10.1207/ S15324796ABM2403_04

Craig, P., Dieppe, P., Macintyre, S., Michie, S., Nazareth, I., \& Petticrew, M. (2013). Developing and evaluating complex interventions: The new Medical Research Council guidance. International Journal of Nursing Studies, 50, 587-592. doi:10.1016/j.ijnurstu.2012.09.010

Craig, P., Dieppe, P., Macintyre, S., Michie, S., Nazareth, I., \& Petticrew, M.; Medical Research Council Guidance. (2008). Developing and evaluating complex interventions: The new Medical Research Council guidance. BMJ (Clinical Research ed.), 337, a1655. doi:10.1136/bmj.a1655

Cruz-Jentoft, A. J., Baeyens, J. P., Bauer, J. M., Boirie, Y., Cederholm, T., Landi, F.,...Zamboni, M.; European Working Group on Sarcopenia in Older People. (2010). Sarcopenia: European consensus on definition and diagnosis: Report of the European Working Group on Sarcopenia in Older People. Age and Ageing, 39, 412-423. doi:10.1093/ageing/afq034

Cruz-Jentoft, A. J., Landi, F., Schneider, S. M., Zúñiga, C., Arai, H., Boirie, Y.,...Cederholm, T. (2014). Prevalence of and interventions for sarcopenia in ageing adults: A systematic review. Report of the International Sarcopenia Initiative (EWGSOP and IWGS). Age and Ageing, 43, 748-759. doi:10.1093/ageing/ afu115

Cullen, K. W., Baranowski, T., \& Smith, S. P. (2001). Using goal setting as a strategy for dietary behavior change. Journal of the American Dietetic Association, 101, 562-566. doi:10.1016/ S0002-8223(01)00140-7

Daly, R. M., O’Connell, S. L., Mundell, N. L., Grimes, C. A., Dunstan, D. W., \& Nowson, C. A. (2014). Protein-enriched diet, with the use of lean red meat, combined with progressive resistance training enhances lean tissue mass and muscle strength and reduces circulating IL- 6 concentrations in elderly women: A cluster randomized controlled trial. The American Journal of Clinical Nutrition, 99(4), 899-910. doi:10.3945/ ajcn.113.064154

Dedeyne, L., Dewinter, L., Lovik, A., Verschueren, S., Tournoy, J., \& Gielen, E. (2018). Nutritional and physical exercise programs for older people: Program format preferences and (dis)incentives to participate. Clinical Interventions in Aging, 13, 1259-1266. doi:10.2147/CIA.S159819

Devereux-Fitzgerald, A., Powell, R., Dewhurst, A., \& French, D. P. (2016). The acceptability of physical activity interventions to older adults: A systematic review and meta-synthesis. Social Science \& Medicine (1982), 158, 14-23. doi:10.1016/j. socscimed.2016.04.006

Durlak, J. A., \& DuPre, E. P. (2008). Implementation matters: A review of research on the influence of implementation on program outcomes and the factors affecting implementation. American Journal of Community Psychology, 41, 327-350. doi:10.1007/ s10464-008-9165-0 
Fried, L. P., Tangen, C. M., Walston, J., Newman, A. B., Hirsch, C., Gottdiener, J.,...McBurnie, M. A.; Cardiovascular Health Study Collaborative Research Group. (2001). Frailty in older adults: Evidence for a phenotype. The Journals of Gerontology, Series A: Biological Sciences and Medical Sciences, 56, M146-M156. doi:10.1093/gerona/56.3.m146

Greaves, C. J., Sheppard, K. E., Abraham, C., Hardeman, W., Roden, M., Evans, P. H.,...Schwarz, P.; IMAGE Study Group. (2011). Systematic review of reviews of intervention components associated with increased effectiveness in dietary and physical activity interventions. BMC Public Health, 11, 119. doi:10.1186/1471-2458-11-119

Hardy, S., \& Grogan, S. (2009). Preventing disability through exercise: Investigating older adults' influences and motivations to engage in physical activity. Journal of Health Psychology, 14, 1036-1046. doi:10.1177/1359105309342298

Herrema, A. L., Westerman, M. J., van Dongen, E. J. I., Kudla, U., \& Veltkamp, M. (2018). Combined protein-rich diet with resistance exercise intervention to counteract sarcopenia: A qualitative study on drivers and barriers of compliance. Journal of Aging and Physical Activity, 26, 106-113. doi:10.1123/ japa.2017-0126

Host, A., McMahon, A. T., Walton, K., \& Charlton, K. (2016). Factors influencing food choice for independently living older people-A systematic literature review. Journal of Nutrition in Gerontology and Geriatrics, 35, 67-94. doi:10.1080/21551197 .2016.1168760

Hou, L., Lei, Y., Li, X., Huo, C., Jia, X., Yang, J.,...Wang, X.-M. (2019). Effect of protein supplementation combined with resistance training on muscle mass, strength and function in the elderly: A systematic review and meta-analysis. The Journal of Nutrition, Health \& Aging, 23, 451-458. doi:10.1007/s12603-019-1181-2

Linnan, L., \& Steckler, A. (2002). Process evaluation for public health interventions and research: An overview. In A. Steckler \& L. Linnan (Eds.), Process evaluation for public health interventions and research (1st ed., pp. 1-23). San Francisco: Jossey Bass.

Lutomski, J. E., Baars, M. A. E., Schalk, B. W. M., Boter, H., Buurman, B. M., den Elzen, W. P.,...Melis, R. J.; TOPICS-MDS Consortium. (2013). The development of the Older Persons and Informal Caregivers Survey Minimum DataSet (TOPICS-MDS): A large-scale data sharing initiative. PLoS ONE, 8(12), e81673. doi:10.1371/journal.pone.0081673

Moore, G. F., Audrey, S., Barker, M., Bond, L., Bonell, C., Hardeman, W.,...Baird, J. (2015). Process evaluation of complex interventions: Medical Research Council guidance. BMJ (Clinical Research ed.), 350, h1258. doi:10.1136/bmj.h1258

Morley, J. E., Baumgartner, R. N., Roubenoff, R., Mayer, J., \& Nair, K. S. (2001). Sarcopenia. The Journal of Laboratory and Clinical Medicine, 137, 231-243. doi:10.1067/mlc.2001.113504

Nutbeam, D. (1998). Evaluating health promotion-Progress, problems and solutions. Health Promotion International, 13(1), 27-44. doi:10.1093/heapro/13.1.27

O’Brien, N., McDonald, S., Araújo-Soares, V., Lara, J., Errington, L., Godfrey, A.,...Sniehotta, F. F. (2015). The features of interventions associated with long-term effectiveness of physical activity interventions in adults aged 55-70 years: A systematic review and meta-analysis. Health Psychology Review, 9, 417433. doi:10.1080/17437199.2015.1012177
Olanrewaju, O., Kelly, S., Cowan, A., Brayne, C., \& Lafortune, L. (2016). Physical activity in community dwelling older people: A systematic review of reviews of interventions and context. PLoS ONE, 11, e0168614. doi:10.1371/journal.pone.0168614

Petrescu-Prahova, M., Belza, B., Kohn, M., \& Miyawaki, C. (2016). Implementation and maintenance of a community-based older adult physical activity program. The Gerontologist, 56, 677686. doi:10.1093/geront/gnv024

Proctor, E., Silmere, H., Raghavan, R., Hovmand, P., Aarons, G., Bunger, A.,...Hensley, M. (2011). Outcomes for implementation research: Conceptual distinctions, measurement challenges, and research agenda. Administration and Policy in Mental Health, 38, 65-76. doi:10.1007/s10488-010-0319-7

Proctor, E. K., Landsverk, J., Aarons, G., Chambers, D., Glisson, C., \& Mittman, B. (2009). Implementation research in mental health services: An emerging science with conceptual, methodological, and training challenges. Administration and Policy in Mental Health, 36, 24-34. doi:10.1007/s10488-008-0197-4

Rogers, E. (2003). Diffusion of innovation. New York: Free Press.

Salter, K. L., \& Kothari, A. (2014). Using realist evaluation to open the black box of knowledge translation: A state-ofthe-art review. Implementation Science, 9, 115. doi:10.1186/ s13012-014-0115-y

Saunders, R. P., Evans, M. H., \& Joshi, P. (2005). Developing a process-evaluation plan for assessing health promotion program implementation: A how-to guide. Health Promotion Practice, 6, 134-147. doi:10.1177/1524839904273387

Thomas, D. R. (2006). A general inductive approach for analyzing qualitative evaluation data. American Journal of Evaluation, 27(2), 237-246. doi:10.1177/1098214005283748

Tieland, M., Dirks, M. L., van der Zwaluw, N., Verdijk, L. B., van de Rest, O., de Groot, L. C. P. G. M., \& van Loon, L. J. C. (2012). Protein supplementation increases muscle mass gain during prolonged resistance-type exercise training in frail elderly people: A randomized, double-blind, placebo-controlled trial. Journal of the American Medical Directors Association, 13(8), 713-719. doi:10.1016/j.jamda.2012.05.020

van Dongen, E. J. I., Haveman-Nies, A., Doets, E., Dorhout, B., \& De Groot, C. (2020). Effectiveness of a diet and resistance exercise intervention on muscle health in older adults: ProMuscle in Practice. Journal of the American Medical Directors Association. doi:10.1016/j.jamda.2019.11.026

van Dongen, E. J. I., Haveman-Nies, A., Wezenbeek, N. L. W., Dorhout, B. G., Doets, E. L., \& de Groot, L. C. P. G. M. (2018). Effect, process, and economic evaluation of a combined resistance exercise and diet intervention (ProMuscle in Practice) for community-dwelling older adults: Design and methods of a randomised controlled trial. BMC Public Health, 18, 877. doi:10.1186/s12889-018-5788-8

van Dongen, E. J. I., Leerlooijer, J. N., Steijns, J. M., Tieland, M., de Groot, L. C., \& Haveman-Nies, A. (2017). Translation of a tailored nutrition and resistance exercise intervention for elderly people to a real-life setting: Adaptation process and pilot study. BMC Geriatrics, 17, 25. doi:10.1186/s12877-017-0413-8

Vikberg, S., Sörlén, N., Brandén, L., Johansson, J., Nordström, A., Hult, A., \& Nordström, P. (2019). Effects of resistance training on functional strength and muscle mass in 70-year-old individuals with pre-sarcopenia: A randomized controlled trial. 
Journal of the American Medical Directors Association, 20, $28-$ 34. doi:10.1016/j.jamda.2018.09.011

Wang, S., Moss, J. R., \& Hiller, J. E. (2006). Applicability and transferability of interventions in evidence-based public health. Health Promotion International, 21, 76-83. doi:10.1093/heapro/dai025
Zubala, A., MacGillivray, S., Frost, H., Kroll, T., Skelton, D. A., Gavine, A.,...Morris, J. (2017). Promotion of physical activity interventions for community dwelling older adults: A systematic review of reviews. PLOS ONE, 12, e0180902. doi:10.1371/ journal.pone.0180902 